Life Pure Fusion Target Designs: Status and Prospects

P. Amendt, M. Dunne, D. Ho, J. Lindl

November 14, 2011

Fusion Science and Technology American Nuclear Society 
This document was prepared as an account of work sponsored by an agency of the United States government. Neither the United States government nor Lawrence Livermore National Security, LLC, nor any of their employees makes any warranty, expressed or implied, or assumes any legal liability or responsibility for the accuracy, completeness, or usefulness of any information, apparatus, product, or process disclosed, or represents that its use would not infringe privately owned rights. Reference herein to any specific commercial product, process, or service by trade name, trademark, manufacturer, or otherwise does not necessarily constitute or imply its endorsement, recommendation, or favoring by the United States government or Lawrence Livermore National Security, LLC. The views and opinions of authors expressed herein do not necessarily state or reflect those of the United States government or Lawrence Livermore National Security, LLC, and shall not be used for advertising or product endorsement purposes. 


\title{
LIFE PURE FUSION TARGET DESIGNS: STATUS AND PROSPECTS
}

\author{
Peter Amendt, M. Dunne, D.D. Ho and J.D. Lindl \\ Lawrence Livermore National Laboratory, P.O. Box 808, Livermore CA 94551 \\ Email:amendt1@llnl.gov
}

\begin{abstract}
Analysis and radiation-hydrodynamics simulations for expected high-gain fusion target performance on a demonstration 1-GWe Laser Inertial Fusion Energy (LIFE) power plant are presented. The required laser energy driver is $2.2 \mathrm{MJ}$ at a 0.351-um wavelength, and a fusion target gain greater than 60 at a repetition rate of $16 \mathrm{~Hz}$ is the design goal for economic and commercial attractiveness. A scaling-law analysis is developed to benchmark the design parameter space for hohlraumdriven central hot-spot ignition. A suite of integrated hohlraum simulations is presented to test the modeling assumptions and provide a basis for near-term experimental resolution of the key physics uncertainties on the National Ignition Facility.
\end{abstract}

\section{INTRODUCTION}

The National Ignition Facility (NIF) is poised to demonstrate ignition by 2012 based on the central hotspot (CHS) mode of ignition and propagating thermonuclear burn. ${ }^{1}$ This immediate prospect underscores the imperative and timeliness of advancing inertial fusion as a carbon-free, virtually limitless source of energy by the mid- $21^{\text {st }}$ century to substantially offset fossil fuel technologies. To this end, an intensive effort is under way to leverage success at the NIF and to provide the foundations for a prototype "LIFE.1" engineering test facility, followed by a commercially viable "LIFE.2" demonstration power plant operating at $1 \mathrm{GWe}$. The current design goal for LIFE. 2 is to accommodate $\sim 2.2$ $\mathrm{MJ}$ of laser energy (entering the high- $Z$ radiation enclosure or "hohlraum") at a $0.351-\mu \mathrm{m}$ wavelength operating at a repetition rate of $16 \mathrm{~Hz}$ and to provide a fusion target yield of $132 \mathrm{MJ}$.

To achieve this design goal first requires a " $0-\mathrm{d}$ " analytic gain model that allows convenient exploration of parameter space and target optimization. This step is then followed by 2- and 3-dimensional radiationhydrodynamics simulations that incorporate laser beam transport, x-ray radiation transport, atomic physics, and thermonuclear burn. ${ }^{2}$ These simulations form the basis for assessing the susceptibility to hydrodynamic instability growth, target performance margins, laser backscatter induced by plasma density fluctuations within the hohlraum, and the threat spectrum emerging from the igniting capsule, e.g., spectra, fluences and anisotropy of the $\mathrm{x}$ rays and ions, for input into the chamber survivability calculations. The simulations follow the guidelines of a "point design" methodology, which formally designates a well-defined milestone in concept development that meets established criteria for experimental testing.

In this paper we present in Sec. II the 0-d analytic gain model to survey gain versus laser energy parameter space. Section III looks at the status of integrated hohlraum simulations and the needed improvements in laser-hohlraum coupling efficiency to meet the LIFE.2 threshold (net) target gain of $\sim 60$. Section IV considers advanced hohlraum designs to well exceed the LIFE.2 design goal for satisfactory performance margins. We summarize in Sec. V.

\section{ANALYTIC SCALING LAWS}

Here, we develop some analytical scaling relations to help provide target-design guidance for candidate CHS LIFE. 2 targets. The canonical laser energy for the $3 \omega$ 300-eV National Ignition Campaign (NIC) $\mathrm{CH}$ ablator point design is $1.2 \mathrm{MJ}$, delivered over a duration of $\approx 19$ ns. The capsule absorbs $186 \mathrm{~kJ}$ and produces $15.9 \mathrm{MJ}$ of yield. The hohlraum case-to-capsule radii ratio (CCR), defined as $\sqrt{A_{w} / A_{\text {cap }}}$ where $A_{w}$ is the hohlraum wall area and $A_{\text {cap }}$ the capsule area, is 2.76 , the laser-entrancehole (LEH) fraction is $57 \%$ (by radius) and the capsule radius is $1108 \mu \mathrm{m}$. From energy conservation we write:

$$
\eta P_{L}=\sigma_{B} T_{R}^{4}\left[\left(1-\alpha_{w}\right) A_{W}+2 A_{L E H}+\left(1-\alpha_{c a p}\right) A_{c a p}\right]
$$


where $\eta \cong 0.70 \tau(n s)^{0.13}$ is the hohlraum conversion efficiency (from laser energy to $\mathrm{x}$ rays), ${ }^{1} P_{L}$ is the (peak) laser power, $T_{R}$ is the hohlraum (peak) radiation temperature, $\alpha_{w}$ is the hohlraum wall x-ray albedo, $A_{L E H}$ is the area of one of the two LEHs, $\alpha_{\text {cap }}$ is the capsule $\mathrm{x}$ - ray albedo, and $\tau$ is the duration of the (flattop equivalent) peak power portion of the laser drive pulse. Using the similarity solutions from a Marshak wave analysis, one obtains $1-\left\langle\alpha_{w}\right\rangle=0.52 T_{R}^{-0.7} \tau^{-0.38}$ where angular brackets denote a time average over $\tau$. ${ }^{1}$ We rewrite Eq. (1) as:

$$
P_{L}=\frac{\sigma_{B} T_{R}^{4} 4 \pi R_{c a p}^{2} C C R^{2}}{0.7 \tau^{0.13}}\left[0.52 T_{R}^{-0.7} \tau^{-0.38}+\frac{f_{L E H}^{2}}{1-f_{L E H}^{2}+\frac{2 z_{H}}{R_{H}}}+\frac{\left(1-\alpha_{c a p}\right)}{C C R^{2}},\right]
$$

where $f_{L E H}$ is the LEH fraction (by radius), $R_{H}$ is the (cylindrical) hohlraum radius, $2 z_{H}$ is the hohlraum length and $R_{c a p}$ is the initial capsule radius. Further progress is made by introducing the peak implosion speed: $v_{i m p}$ $[\mu \mathrm{m} / \mathrm{ns}]=5.085 \gamma \beta^{3 / 5} T_{R}^{0.9}[\mathrm{heV}],{ }^{2}$ where $\beta$ is the ratio of the pressure at a given density to the Fermi pressure, $\gamma=R_{\text {cap }} / \Delta$ is the shell in-flight aspect ratio $(\cong 30)$, and the radiation temperature is written in units of $100 \mathrm{eV}$, i.e., hecto-electron Volts or heV. According to Ref. (2),

$$
\begin{gathered}
R_{c a p}[c m]=\frac{\beta^{1 / 5} \gamma^{1 / 3}}{6.325 T_{R}^{1.03}[h e V]} E^{1 / 3}[M J], \\
\tau[n s] \approx \frac{R_{c a p}}{v_{i m p}}=\frac{399 E_{c a p}^{1 / 3}[M J]}{\tau_{R}^{1.93}[h e v] \beta^{2 / 5} \gamma^{2 / 3}},
\end{gathered}
$$

where $E_{\text {cap }}=4 \pi R_{\text {cap }}^{2} \sigma_{B} T_{R}^{4} \tau$ is the absorbed capsule energy. ${ }^{2}$ Using Eqs. (3a-b) in Eq. (2) and defining $E_{\text {laser }} \cong P_{L} \tau$, we obtain:

$$
\begin{aligned}
E_{\text {laser }}[M J]= & 0.842 \cdot T_{R}^{0.25}[\mathrm{heV}] \cdot \beta^{0.052} \gamma^{0.0867} E_{\text {cap }}^{0.956}[M J] \cdot C C R^{2} \times \\
& {\left[\begin{array}{l}
\left.0.0544 T_{R}^{0.0345}[\mathrm{heV}] \cdot E_{\text {cap }}^{-0.127}[M J] \cdot \gamma^{0.253} \beta^{0.152}+\frac{f_{L E H}^{2}}{1-f_{L E H}^{2}+\frac{2 z_{H}}{R_{H}}+\frac{\left(1-\alpha_{c a p}\right)}{C C R^{2}}}\right]
\end{array}\right.}
\end{aligned}
$$

Note that $E_{\text {laser }}$ scales almost linearly with $E_{\text {cap }}$, but the dependence on $T_{R}$ is fairly weak. The capsule absorbed energy scales with thermonuclear yield $Y$ as:

$$
E_{c a p}[M J]=0.0285 \cdot Y^{3 / 5}[M J]
$$

based on radiations-hydrodynamics simulations. ${ }^{2}$ The amount of fuel mass $m_{f}$ at the instant of peak implosion velocity scales as $E_{\text {cap }} / v_{\text {imp }}^{2}$, assuming most of the remaining shell mass is in fuel. Thus, more thermonuclear yield is possible for lower implosion speeds at fixed $E_{c a p}$, subject to the constraint of a preserved performance margin $M \propto R_{\text {cap }}^{3} v_{i m p}^{7.7} \cong E_{c a p} v_{i m p}^{8.7} \cdot{ }^{3}$ At fixed margin the amount of available fuel mass now scales more favorably: $m_{f} \propto E_{\text {cap }}^{10.7 / 8.7}$ instead of $\propto E_{\text {cap }}$, which in turn gives in place of Eq. (5), after ignoring the slight increase in burn fraction with $E_{\text {cap }}$ :

$$
E_{c a p} \propto\left(Y^{3 / 5}\right)^{8.7 / 10.7} \approx Y^{0.49}
$$


The minimum radiation temperature required for ignition with margin $M$ at a given $E_{c a p}$ scales as:

$T_{R}[$ heV $]=66.2 E_{\text {cap }}^{-1 / 5.31}[M J] \cdot M^{-1 / 5.31} \gamma^{-1.11}$,

where the factor of 5.31 in the exponents arises from the scaling of Herrmann et al. for the threshold ignition (absorbed capsule) energy with implosion velocity $\left(v_{i m p}^{-5.9}\right) .{ }^{4}$ Using Eqs. $(6,7)$ to eliminate $E_{\text {cap }}$ and $T_{R}$ in Eq. (2) gives $E_{\text {laser }}$ as a function of $Y$, which we further study. The low energy endpoint is normalized to the NIC $\mathrm{CH}$ point design whose properties have been summarized above. An improvement in hohlraum efficiency at lower drive temperatures has been predicted by Suter et al. based on 1-d hohlraum simulation studies, ${ }^{5}$ and is conveniently parameterized as follows:

$$
E_{\text {laser }} \rightarrow E_{\text {laser }} \cdot\left\{\frac{0.315-0.162 \cdot\left(T_{R}[\mathrm{heV}] / 3\right)^{4}}{0.315-0.162}\right\} \text {. }
$$

Figure 1 shows the expected target gain $\mathrm{G}=\mathrm{Y} / E_{\text {laser }}$ versus $Y$ and $E_{\text {laser }}$ under two scenarios: (1) added fuelmass scaling at fixed margin [Eq. (6)], higher hohlraum efficiency at lower $T_{R}$ [Eq. (8)] (upper curve); and (2) added fuel-mass scaling, but using the nominal hohlraum efficiency (lower curve). The area between the two curves reflects the uncertainty in overall hohlraum efficiency with the lower curve representing a conservative bound.

\section{NIC-LIKE HOHLRAUM SIMULATIONS}

Figure 1 provides a first estimate on the target gains accessible for a given laser energy, subject to assumptions on the laser-to-hohlraum coupling efficiency. The next step is to perform integrated hohlraum simulations that incorporate the physical processes of laser energy propagation and absorption, conversion of laser energy to $\mathrm{x}$ rays, atomic physics, thermal transport radiation transport, and thermonuclear burn.

The key element introduced by integrated, 2-d hohlraum simulations is the requirement for a quasisymmetric capsule implosion. To this end, the hohlraum length is adjusted to provide time-integrated $\mathrm{x}$-ray flux symmetry near hohlraum target center, and the relative laser power between the inner $\left(30^{\circ}\right.$ relative to the hohlraum symmetry axis) and outer $\left(50^{\circ}\right)$ laser cones are temporally adjusted to provide sufficient time-dependent drive symmetry as well. ${ }^{6}$ Figure 1 shows the result of a hohlraum tune in a NIC-like laser and target geometry extrapolated to an earlier first-generation LIFE tune that results in a nearly symmetric capsule implosion. The capsule used in the simulation is a high-density carbon ablator that absorbs $\sim 770 \mathrm{~kJ}$ of $\mathrm{x}$ rays, is driven at $\sim 250 \mathrm{eV}$ peak hohlraum temperature, is designed to have similar performance margin as the NIC $\mathrm{CH}$ capsule tune, and has a fuel burn-up fraction of nearly $30 \%$. The high-density carbon ablator has the added advantages of high material strength for survival against target injection stresses and subsequent threats from the harsh target chamber environment. The LEH fraction is $50 \%$ (by radius) (see Fig. 2) instead of the $57 \%$ LEH fraction adopted for the NIC point design.

The required laser energy and resulting target gain is only marginally attractive as a LIFE fusion engine, and

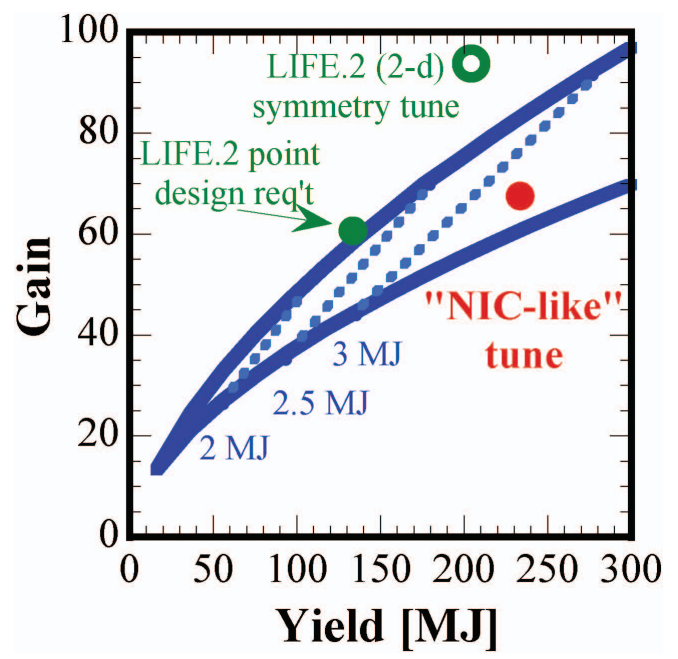

Fig. 1: Gain versus yield scaling for several values of laser energy (dotted lines). Top (lower) curve is with (without) improved hohlraum efficiency prediction (Suter et $a .^{5}{ }^{5}$ ). Right-filled circle denotes $E_{\text {cap }}=3.45 \mathrm{MJ}$ integrated (cylindrical) hohlraum symmetry tune with NIClike beam geometry; left-filled circle is LIFE.2 design point $\left(E_{\text {laser }}=2.17 \mathrm{MJ}\right.$ at $\left.\mathrm{LEH}, Y=205 \mathrm{MJ}\right)$; open circle is symmetry tune from a tuned 2-d integrated hohlraum calculation.

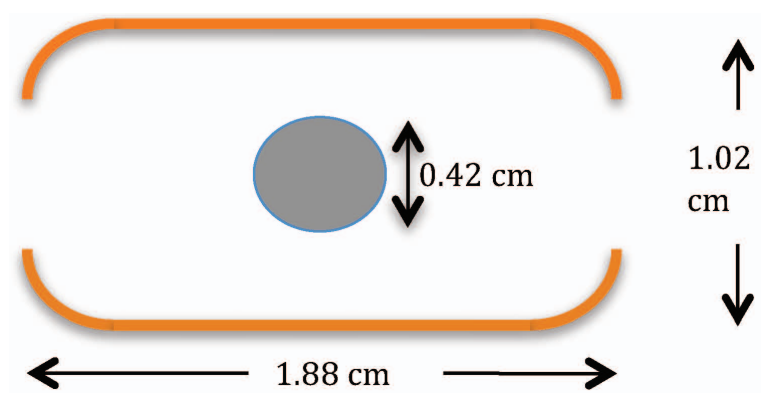

Fig. 2: Schematic of NIC-like hohlraum symmetry tune requiring $3.45 \mathrm{MJ}$ of $3 \omega$ laser energy and giving a gain $\sim 67$; LEH fraction is $50 \%$. 
recent work has focused on the more economically viable LIFE. 2 point design. ${ }^{7}$ Figure 1 shows that this design point lies directly on the upper (high efficiency) curve for hohlraum coupling, but requires only a modest improvement in coupling efficiency of $\sim 11 \%$ over the earlier NIC-like tune. Figure 1 shows the status of an optimized hohlraum symmetry tune that well exceeds the LIFE. 2 design goal of gain $\sim 60$. These simulations use a NIC-like hohlraum made of $\mathrm{Au} / \mathrm{U}$ for maximizing the degree of coupling efficiency to the capsule. A LIFE.2 hohlraum will require far more plentiful materials for cost competitiveness, ${ }^{8}$ such as $\mathrm{Pb}$. Simulations directly comparing the efficiency between a NIC-like hohlraum and a LIFE. 2 hohlraum based on pure $\mathrm{Pb}$ show a modest several percentage deficit in coupling $\mathrm{x}$-ray energy to the capsule, requiring in turn slightly more laser energy for a LIFE. 2 hohlraum. In addition, DT fuel loading of a LIFE. 2 capsule will require an alternative to the current timeconsuming process of $\beta$-layering that is used by the NIC. The application of low-density nanoporous $20-30 \mathrm{mg} / \mathrm{cc}$ $\mathrm{CH}_{1.2}$ annular foams for supporting liquid DT fuels could provide a more cost-effective means for massmanufacturing LIFE.2 targets and a reduced tritium inventory, but with a potential $\sim 10 \%$ penalty in gain degradation. Additional performance penalties are likely to result from hydrodynamic (interface) mix, plasmamediated laser backscatter, laser mispointings and hohlraum misorientations. The current $\sim 55 \%$ gain margin in "clean" 2-d integrated hohlraum simulations, i.e., 94 versus the LIFE. 2 net gain requirement of 60 , is intended to provide sufficient margin to such performance errors and the degradations expected from the above-required fabrication strategies. Methods to offset these errors and degradations are based largely on changes to the hohlraum geometry to improve the laser-hohlraum coupling efficiency as described below.

\section{ADVANCED HOHLRAUM CONCEPTS}

For CHS ignition, the NIC-like hohlraum geometry is currently deemed too marginal to pursue further as an economically defensible candidate for LIFE.2. Target design improvements and forthcoming experimental results on the NIF may favorably alter this status, but a dedicated effort to significantly increase the hohlraum coupling efficiency by a number of methods is a prudent course in the interim.

To overcome the expected drive deficit with $\mathrm{Pb}$ hohlraums and foam-supported DT fuel loading and to provide sufficient performance margin to plasma-initiated laser backscatter, target fabrication, laser engagement errors, and expected yield degradation from hydrodynamic (interface) instabilities, geometry improvements to the
NIC-like hohlraum configuration are sought. Three types of advances in hohlraum design are envisioned: (1) rugbyshaped hohlraums for reduced wall energy losses, (2) high$Z$, axial shields on the hohlraum symmetry axis to block the capsule view of the (lossy) LEHs, and (3) a reduced CCR for higher $E_{\text {cap }}$ (see Fig. 3). The use of rugby-shaped hohlraums reduces the hohlraum wall surface area by nearly $30 \%$ for the case of $50 \%$ LEHs, translating into a potential $15-20 \%$ savings in required laser energy. The rugby concept enjoys confirming experimental evidence to date, where a $\sim 20 \%$ improvement in flux on capsule for the case of vacuum hohlraums was recently demonstrated. ${ }^{9}$ Axial shields increase the flux on the capsule by $10-15 \%$, according to radiation-hydrodynamics simulations. The effects of axial discs on symmetry and drive in vacuum hohlraums were validated by experiments on the Nova laser. ${ }^{10}$ A 5\% larger capsule relative to the hohlraum radius can provide another $\sim 15 \%$ in $E_{\text {cap }}$, provided flux symmetry control is not adversely affected. These three improvements together total $\sim 47 \%$ in increased hohlraum coupling efficiency according to hohlraum simulations, easily overcoming the deficit from the use of $\mathrm{Pb}$ hohlraums and foam-supported DT fuel loading, as well as providing needed performance margin for LIFE.2.

However, testing of these design elements, both individually and collectively, on the NIF over the near term will help define the physical limits of their integrated use for LIFE.2. Ultimately, testing of LIFE-relevant hohlraums on the NIF over the next several years will refine the allowable performance margins for LIFE. 2 and tightly constrain the requirements for target fabrication and robustness to injection stresses and laser engagement errors.

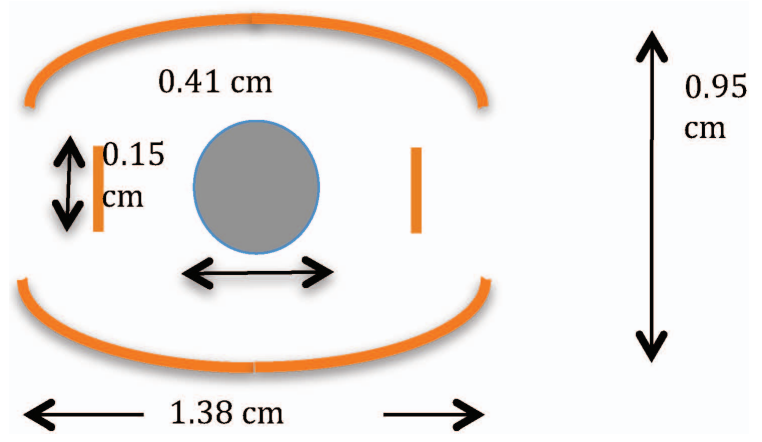

Fig. 3: Rugby-shaped hohlraum version of NIC-like LIFE point design, cf., Fig. 1, with 0.15 -cm-diameter axial shields and 50\% LEHs. Required laser energy is $\sim 2.17 \mathrm{MJ}$ and target gain is $\sim 94$. 


\section{SUMMARY}

The availability of the NIF for near-term ignition experiments provides a critical and timely rationale for a serious pursuit of inertial-fusion-energy commercial strategies, such as LIFE.2. Many of the outstanding physics challenges and questions are amenable to direct testing on the NIF, forestalling the need for intermediate and transitional test facilities. In particular, the fusion engine proposed for a LIFE. 2 power plant in many respects can be largely vetted on the NIF for specifying performance margins and target manufacturing tolerances.

The general guidelines for the fusion engine driver underlying a cost-effective LIFE. 2 power plant design have been introduced. A minimum target gain of $\sim 60$ at an input laser energy driver of $\sim 2.2 \mathrm{MJ}$ (delivered to the hohlraum) is the working assumption. Advanced hohlraum concepts using rugby shapes, axial discs and a reduced CCR are planned in tandem to well exceed this goal, allowing for significant margin to performance degrading target imperfections, laser backscatter and non-ideal target engagement by the lasers. More study is planned to optimally balance the requirements for high-gain (static) target performance with robustness to chamber insults incurred by a transiting fusion target.

\section{ACKNOWLEDGMENTS}

This work performed under the auspices of the U.S. Department of Energy by Lawrence Livermore National Laboratory under Contract DE-AC52-07NA27344.

\section{REFERENCES}

1. J.D. LINDL et al., Phys. Plasmas 11 (2), 339 (2004).

2. J.D. LINDL, Inertial Confinement Fusion (SpringerVerlag, NY, 1998).

3. D.S. CLARK, S.W. HAAN and J.D. SALMONSON Phys. Plasmas 15, 056305 (2008).

4. M.C. HERRMANN, M. TABAK and J.D. LINDL, Nucl. Fusion 4199 (2001).

5. L.J. SUTER, S. HAAN and M. HERRMANN, Bull. Am. Phys. Soc. 50(8), 187 (2005).

6. LIFE. 1 and LIFE. 2 will utilize a simplified cone geometry compared with the NIF of two cones of angles per side of $30^{\circ}$ and $50^{\circ}$ relative to the target chamber vertical axis.

7. M. DUNNE et al., "Timely delivery of Laser Inertial Fusion Energy (LIFE)," these Proceedings; T. Anklam, A. Simon, W. Meier, and S. Powers, "The Case for Early Commercialization of Fusion Energy," these Proceedings.

8. R. MILES et al., "Challenges Surrounding the Injection and Arrival of Targets at LIFE Target Chamber Center," these Proceedings.

9. F. PHILIPPE et al., Phys. Rev. Lett. 104, 035004 (2010); H.F. Robey et al., Phys. Plasmas 17, 056313 (2010).

10. P. AMENDT, T.J. MURPHY and S.P. HATCHETT, Phys. Plasmas 3, 4166 (1996); P. AMENDT, S.G. GLENDINNING, B. HAMMEL, O.L. LANDEN and L.J. SUTER, Phys. Rev. Lett. 77, 3815 (1996); D.A. CALLAHAN, P. AMENDT, E.L. DEWALD, et al., Phys. Plasmas 13, 056307 (2006). 